\title{
ESTUDO DA SUSTENTABILIDADE AGRÍCOLA EM UM MUNICÍPIO AMAZÔNICO COM ANÁLISE ENVOLTÓRIA DE DADOS
}

\author{
Eliane Gonçalves Gomes* \\ Empresa Brasileira de Pesquisa Agropecuária (Embrapa) \\ Brasília - DF \\ eliane.gomes@embrapa.br
}
João Carlos Correia Baptista Soares de Mello
Departamento de Engenharia de Produção
Universidade Federal Fluminense (UFF)
Niterói - RJ
jcsmello@producao.uff.br

\author{
João Alfredo de Carvalho Mangabeira \\ Embrapa Monitoramento por Satélite \\ Campinas - SP \\ manga@cnpm.embrapa.br
}

* Corresponding author / autor para quem as correspondências devem ser encaminhadas

Recebido em 08/2007; aceito em 12/2008 após 1 revisão

Received August 2007; accepted December 2008 after one revision

\begin{abstract}
Resumo
O conceito de sustentabilidade agrícola considera diferentes dimensões. Entretanto, tem como base a valorização dos recursos internos dos sistemas agrícolas produtivos, que se traduz pela manutenção desses sistemas de produção e, consequentemente, de sua produtividade, ao longo do tempo. Este artigo tem como objetivo medir a sustentabilidade de produtores agrícolas a partir de modelos Análise de Envoltória de Dados (Data Envelopment Analysis - DEA) com restrições aos pesos. Nesta abordagem, além de considerar várias dimensões, a medida de eficiência resultante é calculada de forma relativa. Foi usado um modelo no qual se explora o desempenho econômico-ambiental (variáveis: área total, mão-de-obra, custo, receita bruta, área com mata), para dois períodos de tempo, 1986 e 2002.
\end{abstract}

Palavras-chave: DEA; restrições aos pesos; sustentabilidade; agricultura.

\begin{abstract}
The definition of agriculture sustainability considers different aspects. Their main values are the productive systems' internal resources, as they are the way to maintain these systems and, consequently, its productivity through time. In this paper we measure rural producers' sustainability with Data Envelopment Analysis models (DEA) with weights restrictions. This approach considers multiple dimensions and is a relative measure of efficiency. We used a model in which we explore the economic-environmental performance (variables: total area, employment, costs, net income, forested area), in two periods of time, the years of 1986 and 2002.
\end{abstract}

Keywords: DEA; weights restrictions; sustainability; agriculture. 


\section{Introdução}

O desenvolvimento sustentável é objeto de debate há mais de três décadas, com a existência de diversas definições. A definição oficial foi apresentada em 1987, pela Comissão Mundial sobre Meio Ambiente e Desenvolvimento das Nações Unidas no documento "Nosso Futuro Comum" (Comissão Mundial sobre Meio Ambiente e Desenvolvimento, 1988). Segundo esse documento, desenvolvimento sustentável é a busca do atendimento das necessidades presentes sem comprometer as possibilidades de as gerações futuras atenderem suas próprias necessidades.

Sobre a gestão de recursos naturais, segundo López-Ridaura et al. (2002), o desafio é entender e avaliar o desempenho de sistemas socioambientais complexos. Há a necessidade de traduzir os princípios gerais da sustentabilidade em ações práticas. Em agricultura, os mesmos autores avaliam que o objetivo de implementar a sustentabilidade é consolidar princípios complexos e diversos, e transforma-los em recomendações para a tomada de decisão dos agricultores.

Nas atividades agrícolas há diversas formas de conceituar sustentabilidade. Entretanto, embora sejam consideradas dimensões diferentes de um conceito para outro, a base da definição de sustentabilidade em agricultura é a valorização dos recursos internos dos sistemas agrícolas produtivos (Edwards et al., 1990). Sobre o tema "agricultura sustentável” citam-se, por exemplo, os trabalhos de Veiga (1996), Ehlers (1999), Von Wirén-Lehr (2001) e Pretty (2008).

A sustentabilidade dos sistemas agrícolas de produção traduziu-se, historicamente, pela manutenção da produtividade ao longo do tempo. Como evolução desse conceito, foram acrescidas ideias de estabilidade da produtividade e de equidade dos sistemas de produção (medida pela distribuição estatística de parâmetros como renda, número de propriedades, população etc.). Na última década, a sustentabilidade surgiu como a característica a ser analisada e medida (Carpenter, 1993). Na agricultura, a questão da sustentabilidade envolve fatores físicos, bióticos e aspectos relativos à viabilidade econômica e sociocultural.

No caso brasileiro, iniciativas de desenvolvimento agrícola sustentável espalham-se pelo país, em diversas condições agroambientais e socioeconômicas. No entanto, a avaliação de seu desempenho enfrenta as dificuldades impostas pela grande complexidade de interrelações das variáveis do meio físico, de uso e ocupação das terras e de fatores socioeconômicos. Sachs $(1990,2000)$ destaca que as dimensões principais para avaliar a sustentabilidade são: social, cultural, ecológica, ambiental e econômica.

Na literatura há diversas abordagens para avaliar a sustentabilidade agrícola. A maioria propõe a construção de indicadores multidimensionais, que podem ou não ser agregados em um "índice de sustentabilidade" único, que avalie o desempenho do sistema produtivo. Citam-se nesta abordagem os trabalhos de, por exemplo, Ali (1996), Toresan (1998), Marzall \& Almeida (2000), Pannell \& Glenn (2000), Rigby et al. (2001), Praneetvatakul et al. (2001), López-Ridaura et al. (2002), Herendeen \& Wildermuth (2002), Lopes \& Almeida (2003), Pacini et al. (2003), Zhen \& Routray (2003), Fernandes (2004), Gomes (2004), Rasul \& Thapa (2004), Parra-Lopez et al. (2008). No caso específico do acompanhamento e a avaliação da evolução da sustentabilidade e do desempenho de projetos de colonização agrícola ou de assentamentos rurais, citam-se as pesquisas de Melgarejo (2000) e Barreto et al. (2005).

Este artigo tem como objetivo medir a sustentabilidade de produtores agrícolas. Foram usados modelos Análise Envoltória de Dados (Data Envelopment Analysis - DEA). Essa foi a abordagem escolhida, pois a medida de eficiência resultante é calculada de forma comparada (relativa) e permite considerar várias dimensões. Foi usado um modelo no qual se explora o desempenho econômico-ambiental (variáveis: área total, mão-de-obra, custo, 
receita bruta, área com mata). Esse modelo foi usado para avaliar a sustentabilidade de um grupo de produtores rurais do município de Machadinho d'Oeste (RO). Como a sustentabilidade agrícola preconiza a manutenção dos sistemas de produção ao longo do tempo, os modelos foram rodados para dois períodos de tempo, 1986 e 2002.

A dimensão social não está explícita neste modelo. Esta foi apropriadamente estudada por Gomes et al. (2008), onde, com uso de modelos multicritério e para um período de tempo que contempla o do estudo aqui desenvolvido, mostrou-se que há um aumento do bem estar social rural na região.

\section{Estudo de caso: caracterização da área de estudo}

Machadinho d'Oeste é um município de Rondônia, Amazônia brasileira, localizado entre as coordenadas geográficas $61^{\circ} 47^{\prime}$ e $63^{\circ} 00^{\prime}$ de longitude WGr e $9^{\circ} 19^{\prime}$ e $10^{\circ} 00^{\prime}$ de latitude $S$, distanciado a cerca de $400 \mathrm{~km}$ da capital Porto Velho.

Antes de ser elevado à condição de município em 1988, Machadinho d'Oeste surgiu como um projeto de assentamento rural. Ao ser instituído como município, seus limites foram ampliados e novas áreas foram incorporadas (quatro outros projetos de colonização e oito centros urbanos), em um total de $8.556 \mathrm{~km}^{2}$. Estudos que contemplam diferentes aspectos desta região podem ser vistos, por exemplo, em Moran (1984), Sydenstricker \& Torres (1991), Castilla \& Sawyer (1993), Dale et al. (1994), Charlwood et al. (1996), Souza-Santos (2002).

O projeto de assentamento Machadinho foi dimensionado inicialmente para um total de 3.000 famílias de colonos. Mais de 2.000 já haviam chegado em 1984. Nesse ano, esse projeto de assentamento já tinha infraestrutura mínima para a colonização agrícola, como estradas, núcleos urbanos de apoio, projeto fundiário implantado, lotes demarcados etc.

A partir da implementação do assentamento no início dos anos de 1980, a paisagem original tem sido transformada pelos colonos, em um mosaico composto por remanescentes florestais, vegetação secundária, pastagens, culturas agrícolas e pequenas áreas urbanizadas. Em Batistella et al. (2000, 2003) e Batistella (2001) encontram-se estudos sobre as mudanças da paisagem e do uso e cobertura das terras em Machadinho d'Oeste.

Em 1986 foi definido um projeto de pesquisa para acompanhar os sistemas de produção agrícola praticados por pequenos agricultores desta região (Miranda, 2005). Pesquisas de campo são feitas a cada três anos, com aplicação de questionários com cerca de 250 variáveis agronômicas, ambientais, econômicas e sociais, sobre uma amostra de propriedades. A cada ano, o uso das terras é monitorado por imagens de satélites e espacializado em um Sistema de Informações Geográficas (GIS).

No primeiro ano da pesquisa, 1986, foi realizado um esforço de amostragem que cobriu cerca de $15 \%$ dos lotes, em um total de 438 lotes válidos. Essa amostra aleatória e estratificada foi geocodificada em um GIS. Os resultados do primeiro levantamento de dados in loco permitiram definir um perfil inicial dos agricultores recém chegados e de sua agricultura.

Em 1989, um novo levantamento permitiu elaborar e espacializar em GIS o perfil da agricultura e dos agricultores. Em 1993, outra etapa foi realizada, aprofundando aspectos vinculados à economia e ao meio ambiente. A pesquisa de campo de 1996 mostrou que, em 10 anos, houve mudanças profundas nas propriedades, tanto na estrutura como nos sistemas de produção.

Em um novo levantamento de campo em 1999 foram entrevistados 438 agricultores, cujos sistemas de produção foram novamente caracterizados. Caso a propriedade tivesse mudado

Pesquisa Operacional, v.29, n.1, p.23-42, Janeiro a Abril de 2009 
de dono, o novo responsável era entrevistado e, dessa forma, foi elaborado um novo perfil dos agricultores e da agricultura.

Em setembro e outubro de 2002, um novo levantamento foi conduzido junto às propriedades estudadas em anos anteriores, com a atualização das variáveis sobre os sistemas de produção praticados. Nessa ocasião foram entrevistados 327 agricultores.

Nessas duas décadas de pesquisa, notaram-se mudanças na região, seja em relação aos aspectos econômicos e sociais, seja em relação ao uso da terra. De modo a avaliar a evolução do desempenho desses agricultores, neste artigo foram usados modelos DEA para avaliar sua sustentabilidade. A escolha do município de Machadinho d'Oeste deve-se ao fato de esta ser uma área de colonização agrícola na Amazônia. Pesquisadores da Embrapa Monitoramento por Satélite, da Empresa Brasileira de Pesquisa Agropecuária (Embrapa), idealizaram há 20 anos um projeto de acompanhamento, por 100 anos, de um número significativo de pequenas propriedades rurais nessa região. O monitoramento pelos pesquisadores de uma amostra de produtores rurais produziu (e ainda produz; a última pesquisa de campo foi realizada em 2005) uma série de dados sobre propriedades familiares instaladas em floresta tropical úmida, capazes de gerar indicadores sobre sua sustentabilidade agrícola. Os resultados obtidos por este projeto de pesquisa podem ser encontrados em Miranda (2005). Essas informações, segundo Miranda (2005), podem influenciar políticas públicas para a região, em termos de assistência, pesquisa, financiamento etc.

\section{DEA}

\subsection{Aspectos gerais}

Modelos DEA têm como objetivo calcular a eficiência de unidades produtivas, chamadas de unidades de tomada de decisão ou DMUs (Decision Making Units), conhecendo-se os níveis de recursos empregados e de resultados obtidos. DEA otimiza cada observação individual, de modo a estimar uma fronteira eficiente (linear por partes), composta das unidades que apresentam as melhores práticas dentro da amostra em avaliação (unidades Pareto-Koopmans eficientes). Essas unidades servem como referência ou benchmark para as unidades ineficientes.

Os dois modelos mais conhecidos de DEA são o CCR (Charnes et al., 1978) e o BCC (Banker et al., 1984). O modelo CCR trabalha com retornos constantes à escala. O modelo BCC considera retornos variáveis à escala, ou seja, substitui o axioma da proporcionalidade pelo axioma da convexidade. Tradicionalmente são possíveis duas orientações radiais para esses modelos na busca da fronteira de eficiência: orientação a inputs e orientação a outputs.

Para modelar e interpretar corretamente os resultados de DEA é necessário conhecer as propriedades de seus modelos. Duas das mais importantes são:

- Em qualquer modelo DEA, a DMU que apresentar a melhor relação (output $j$ )/(input $i$ ) será sempre eficiente. Esta propriedade obriga a que na formulação do modelo DEA seja necessário verificar a existência de relação causal entre cada output e cada input. A não observância dessa relação pode conduzir a resultados sem sentido.

- O modelo CCR, que na sua forma fracionária é uma função homogênea de grau zero, tem como propriedade principal a proporcionalidade entre inputs e outputs na fronteira. Isto tem como consequência que o aumento (decremento) na quantidade dos inputs, provocará acréscimo (redução) proporcional no valor dos outputs. 


\subsection{Modelos DEA com restrições aos pesos}

Os modelos DEA clássicos permitem total liberdade na escolha dos pesos que darão o máximo valor de eficiência a uma dada DMU. Essa liberdade é importante na identificação das unidades ineficientes, ou seja, daquelas DMUs que apresentam um baixo desempenho inclusive com seu próprio conjunto de multiplicadores. A flexibilidade na escolha dos pesos é uma das vantagens apontadas à modelagem DEA. Entretanto, os pesos calculados podem ser inconsistentes com o conhecimento em relação aos valores relativos de inputs e outputs. Assim, a incorporação de julgamentos de valor dos especialistas a respeito da importância relativa de cada variável no cálculo das medidas de eficiência surge como uma evolução natural das aplicações de DEA a problemas reais. Ou seja, há a necessidade de introduzir condições além das de não negatividade.

Quando há preferências entre os inputs e/ou outputs por parte dos agentes de decisão, esses julgamentos de valor são incorporados aos modelos DEA por meio de restrições aos pesos (ou multiplicadores) associados aos inputs e/ou aos outputs das unidades avaliadas. Allen et al. (1997) e Thanassoulis et al. (2004) apresentam uma revisão da evolução da incorporação de julgamentos de valor através de restrições aos pesos. Angulo Meza \& Lins (2002) consideram que a adição de restrições aos pesos é uma das técnicas que promovem melhoria de discriminação em DEA, com opiniões subjetivas por parte dos decisores. Adler et al. (2002) também incluem as restrições aos pesos dentro das técnicas de melhora de discriminação em DEA e apresentam vários tipos de restrições. As principais são (Lins \& Angulo Meza, 2000): (a) restrições diretas sobre os multiplicadores; (b) ajuste dos níveis de input-output observados para a captura de julgamentos de valor; (c) restrição a inputs e outputs virtuais.

Um dos tipos de modelos de restrições aos pesos é o modelo de Regiões de Segurança Tipo I (Assurance Region Type I - ARI), conforme definido em Thompson et al. (1990). Em (1) apresenta-se a formulação genérica do modelo BCC com as restrições ARI impostas aos outputs, onde $\alpha_{i}$ e $\beta_{i}$ são constantes especificadas pelo decisor e que, após uma conveniente normalização, refletem os julgamentos de valor sobre a importância relativa entre os inputs $i$ e $i+1$. Este é o modelo usado neste artigo.

$$
\begin{aligned}
& \text { Max } \sum_{j=1}^{s} u_{j} y_{j o}-u_{*} \\
& \text { sujeito a } \\
& \sum_{i=1}^{r} v_{i} x_{i o}=1 \\
& -\sum_{i=1}^{r} v_{i} x_{i k}+\sum_{j=1}^{s} u_{j} y_{j k}-u_{*} \leq 0, \forall k \\
& \alpha_{i} \leq \frac{v_{i}}{v_{i+1}} \leq \beta_{i} \\
& u_{j}, v_{i} \geq 0, \forall j, i \\
& u_{*} \in \Re
\end{aligned}
$$

Se se pretendesse avaliar a importância de cada variável na construção dos outputs e inputs virtuais na formação do alvo de cada DMU, deveriam ser usadas restrições do tipo (c). Este 
tipo de restrição tem como desvantagem a alta probabilidade de ocorrerem inviabilidades nos PPLs de DEA (este assunto é discutido em Lins et al., 2007). Por este motivo preferiu-se usar restrições do tipo ARI, com as variáveis previamente normalizadas para evitar problemas de escala das variáveis (Allen et al., 1997). Deve-se notar ainda que as restrições do tipo ARI exigem menos informações dos decisores, o que facilita seu uso prático.

\subsection{Modelos DEA em agricultura}

O uso DEA para medir a eficiência relativa de unidades produtivas tem-se mostrado bastante atrativo em diversos setores de aplicação. O emprego de modelos DEA em agricultura pode vir a apoiar as decisões dos agricultores (e dos órgãos de fomento, assistência técnica, pesquisa ou de associações de agricultores), ao indicar as fontes de ineficiência e as unidades que podem servir de referência às práticas adotadas.

Diversos autores dedicaram-se a rever o uso de modelos de fronteiras de produção na área agrícola. Citam-se: Battese (1992), Bravo-Ureta \& Pinheiro (1993), Coelli (1995), Thiam et al. (2001), Bravo-Ureta et al. (2007). Estes últimos expandiram a revisão de Thiam et al. (2001) e conduziram pesquisa sobre modelos de fronteiras de produção em nível de fazenda. Dos 569 estudos encontrados, 87 usaram modelos não-paramétricos tipo DEA (muitos usavam mais de uma técnica ou tipo de análise).

Uma pesquisa conduzida entre março e maio de 2007 nas bases de dados Web of Science, Scopus, ProQuest, Compendex, WebSpiris, EbscoHost; WilsonWeb, Agricola, Agronbase, AgEcon Search, CIAT e Scielo retornou 158 referências ao uso de modelos DEA em agricultura. Alguns dos artigos tratam apenas do uso de DEA, mas a maioria combina diversos modelos de fronteiras de produção, estatísticos e econométricos. Foi também feita uma pesquisa na base de Currículos Lattes, do CNPq, para identificar publicações de autores nacionais. Estas eram em um total de 20 publicações, sendo duas em periódicos internacionais e o restante em periódicos nacionais. Não foram contabilizados os artigos publicados em anais de eventos científicos, dissertações de mestrado e teses de doutorado. Uma revisão bibliográfica mais extensa sobre este assunto é encontrada em Gomes (2008).

Apesar da quantidade de referências sobre aplicações de DEA em agricultura, estas são escassas quando se referem ao uso de DEA para medir sustentabilidade agrícola. Neste tema citam-se os trabalhos de Toresan (1998), De Koeijer et al. (2002), Abay et al. (2004), Rodríguez-Díaz et al. (2004), Bosetti \& Locatelli (2006) e Sauer \& Abdallah (2007).

Toresan (1998) usou a medida de eficiência DEA para calcular eficiência técnica de 167 empresas agrícolas brasileiras. Os resultados foram comparados com um índice de sustentabilidade de capacidade produtiva do solo, usando a abordagem Macbeth. Em um segundo modelo usou este índice com output de um novo modelo DEA. Como resultado de seu estudo, o autor argumenta que "o processo de adoção de práticas agrícolas sustentáveis não pode ser compreendido apenas através dos aspectos econômicos envolvidos. Um melhor entendimento do processo decisório dos agricultores, de suas crenças e valores, dos conflitos entre objetivos privados e sociais envolvidos na ocupação e uso do solo agrícola é fundamental ao estabelecimento de estratégias mais eficazes para disseminar as práticas agrícolas sustentáveis e deve ser objeto de novas investigações.”.

Em De Koeijer et al. (2002), índices de eficiência DEA foram usados como medidas de sustentabilidade para um grupo de produtores holandeses de beterraba. Pela abordagem proposta pelos autores, sustentabilidade seria a combinação de eficiência ambiental e 
desempenho econômico. Se os agricultores melhorassem a eficiência técnica, alcançariam simultaneamente os objetivos econômico e ambiental. Dessa forma, o aumento da eficiência ambiental e econômica promoveria a sustentabilidade.

A eficiência no uso de insumos químicos na produção de tabaco de cerca de 300 produtores da Turquia foi avaliada por Abay et al. (2004). Os autores usaram modelos DEA CCR e modelos estatísticos para determinar os fatores que afetaram as medidas de eficiência. Os resultados indicaram relação positiva entre eficiência no uso de insumos e sustentabilidade da agricultura praticada. Sauer \& Abdallah (2007) também se concentraram na produção de tabaco. Estudaram a relação entre eficiência produtiva, biodiversidade e manejo de recursos, para sua produção na Tanzânia. Os resultados mostraram que o aumento da eficiência agrícola produtiva era condição para a sustentabilidade ambiental, o que motiva a gestão sustentável dos recursos florestais.

Rodríguez-Díaz et al. (2004) avaliaram o uso eficiente de água para irrigação em distritos espanhóis. O objetivo dos autores era fomentar o uso sustentável deste recurso. Empregaram o modelo DEA BCC e concluíram que a agricultura intensiva teve valores de eficiência superiores aos da agricultura extensiva.

O incentivo a práticas conservacionistas em parques nacionais italianos foi estudado por Bosetti \& Locatelli (2004). Os autores usaram modelos DEA para avaliar a eficiência relativa de áreas protegidas e dar diretrizes para potenciais melhorias em termos de sustentabilidade desses parques.

\section{Modelagem}

A estruturação de um modelo DEA necessita da definição das unidades a avaliar, das variáveis a constituir o modelo (inputs e outputs) e do modelo DEA adotado (CCR, BCC, entre outros; orientação a inputs, a outputs etc.).

\subsection{DMUs}

O objetivo do modelo DEA aqui proposto é medir sustentabilidade em agricultura. O conceito de sustentabilidade agrícola usado neste artigo refere-se à capacidade do produtor em manter seu sistema de produção ao longo do tempo. Dessa forma, as DMUs do modelo DEA são os produtores rurais amostrados e que foram entrevistados pela pesquisa de campo em Machadinho d'Oeste em 1986 e em 2002. Adicionalmente, foi estudado apenas o grupo de produtores rurais familiares que se mantiveram no lote, em um total de 76 . Ou seja, foram descartados os lotes que foram abandonados ou mudaram de proprietário nesse período de tempo. Isto porque produtores diferentes têm racionalidades agrícolas diferentes, o que poderia interferir na avaliação da sustentabilidade.

\subsection{Variáveis}

A etapa de seleção das variáveis em DEA é de grande importância, pois dela depende a qualidade dos resultados. Um modelo com grande número de variáveis pode acarretar em uma avaliação extremamente benevolente, com várias DMUs 100\% eficientes. Para o caso de avaliação da sustentabilidade agrícola em Machadinho d’Oeste, as variáveis do modelo foram escolhidas dentre aquelas coletadas no questionário de campo. 
Embora existam modelos de seleção automática de variáveis (Senra et al., 2007), estes não foram adequados ao caso em estudo. Tal fato deveu-se à disponibilidade de um grande número de DMUs e à falta de dúvidas dos decisores sobre quais variáveis seriam as mais adequadas. De fato, os técnicos envolvidos no projeto de pesquisa, ao analisarem os questionários aplicados aos agricultores, identificaram imediatamente as variáveis relevantes para o estudo em questão.

O modelo aqui proposto tem como objetivo medir a sustentabilidade sob as óticas econômica e ambiental, ou seja, mede o desempenho econômico-ambiental dos lotes analisados. Para esse modelo, os inputs são: área total do lote (em ha), uso total de mão-de-obra (em dias homem) e custo total (em R\$). Os outputs são receita bruta (R\$) e área total com mata (ha). Na escolha destas variáveis foi levada em conta a necessidade de relação causal entre outputs e inputs. Uma desagregação da área total e das receitas por tipo de cultivo teria duas desvantagens. A primeira seria o aumento do número de variáveis. A outra seria produzir resultados sem sentido, como considerar eficiente uma DMU que tivesse a melhor relação entre receita na cultura do café e área cultivada na cultura do arroz, por exemplo. Ressalte-se que a agregação de variáveis, seja com este mesmo fim, seja para reduzir a complexidade dos modelos, já foi usada na literatura, como por exemplo, em Lins et al. (2003).

Em relação ao uso de mão-de-obra foram considerados os ativos da família entre 15 e 65 anos e que trabalhavam na propriedade, os empregados permanentes e os temporários. O input mão-de-obra foi medido em dias-homem. Em Machadinho d'Oeste 1 ativo agrícola trabalha, em média, 300 dias.

O cálculo do custo considerou somente as culturas para as quais havia informações disponíveis sobre custeio para a região, quais sejam, arroz, milho, feijão (culturas anuais), café, guaraná e cacau (culturas perenes). O custo de produção por cultura foi calculado pela relação [número de hectares plantado * custo médio de produção por hectare]. Não foram levados em conta, por falta de dados, outros itens de custo que não os de custeio. A receita bruta foi dada por [número de sacos produzidos de determinada cultura * preço médio do saco]. Para as culturas do cacau e do guaraná, a receita foi calculada por quilograma produzido destes produtos. Receita total e custo total referem-se, respectivamente, à soma das receitas e custos individuais por cultura (produção agrícola no lote). Tem-se, assim, o quanto o produtor gastou (custo) em todos os produtos em determinado ano e o quanto ele ganhou (receita) de tudo o que foi vendido (ou consumido, como no caso de arroz, milho e feijão, por ter deixado de comprar na cidade estes produtos de autoconsumo). Os dados de custo e de preço foram obtidos durante a pesquisa de campo, com os agentes econômicos envolvidos.

É importante destacar duas questões. Primeiro, em 1986 as culturas perenes (café, cacau e guaraná) não tiveram produção, por terem sido plantadas recentemente (os colonos foram assentados em 1984 e em 1986 as plantas perenes eram novas). Ou seja, para esse ano foram consideradas somente as produções das culturas anuais (arroz, milho e feijão). Sendo assim, a receita líquida da maioria dos produtores para 1986 era negativa. Ressalta-se, entretanto, que os produtores assentados estavam no início da atividade e recebiam algum benefício financeiro do INCRA (Instituto Nacional de Colonização e Reforma Agrária) ou tinham empréstimo bancário para financiar o investimento feito nas culturas perenes. Segundo, os valores de custo médio e de preços (para cálculo das receitas) usados foram os de 2005 para as seis culturas. Mesmo precários, estes eram os únicos valores disponíveis. Esta opção foi feita para que não se incorresse em erros econômicos (mudança de moeda, deflação ou correção pelo dólar para igualar os preços etc.). Dentro do contexto da dificuldade de levantamento de informações sobre os sistemas de produção praticados pelos produtores, este modelo é uma 
aproximação razoável da forma de produção dos sistemas de cultivo praticados em Machadinho d'Oeste, não muito diferente da maioria dos assentamentos agrícolas no Brasil.

A Tabela 1 apresenta resumo estatístico das variáveis selecionadas para a amostra de 76 produtores rurais. É interessante notar a progressiva redução de área com mata. Isto era esperado, já que Machadinho d’Oeste aparece várias vezes em noticiários como um dos municípios da Amazônia com elevados índices de desmatamento. A redução de área com mata em relação à área total dos lotes pode ser igualmente observada na Figura 1. Nesta figura nota-se também, sem necessidade de calcular, a redução da correlação entre área total e área com mata.

Tabela 1 - Estatística descritiva de alguns dados de Machadinho d’Oeste.

\begin{tabular}{lrrrr}
\cline { 3 - 5 } & & \multicolumn{1}{c}{ Média } & Mediana & Desvio padrão \\
\hline \multirow{2}{*}{ Área total (ha) } & 1986 & 44,71 & 45,00 & 10,37 \\
& 2002 & 56,62 & 46,50 & 71,96 \\
\hline \multirow{2}{*}{ Área com mata (ha) } & 1986 & 35,98 & 34,87 & 10,42 \\
& 2002 & 17,40 & 15,00 & 12,48 \\
\hline \multirow{2}{*}{ Mão-de-obra } & 1986 & 935,53 & 600,00 & 921,69 \\
(dias homem) & 2002 & $1.322,37$ & $1.200,00$ & 831,64 \\
\hline \multirow{2}{*}{ Custo total (R\$) } & 1986 & $1.117,41$ & 983,00 & 755,89 \\
\hline \multirow{2}{*}{ Receita bruta (R\$) } & 2002 & 871,65 & 520,50 & 878,54 \\
& 1986 & $2.012,33$ & 417,30 & $3.417,60$ \\
& 2002 & $5.477,81$ & $2.760,80$ & $8.605,04$ \\
\hline
\end{tabular}

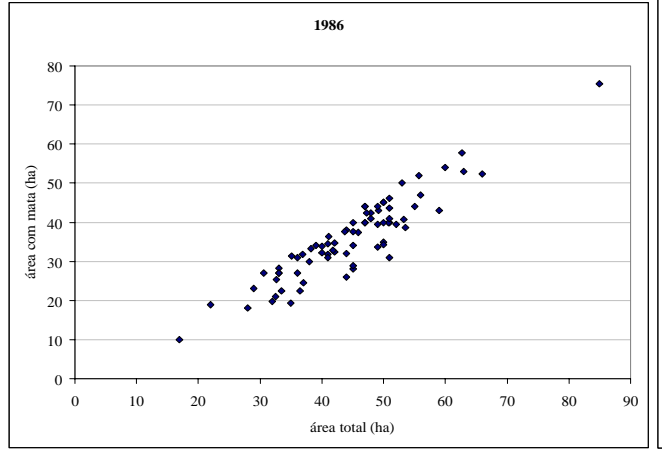

(a)

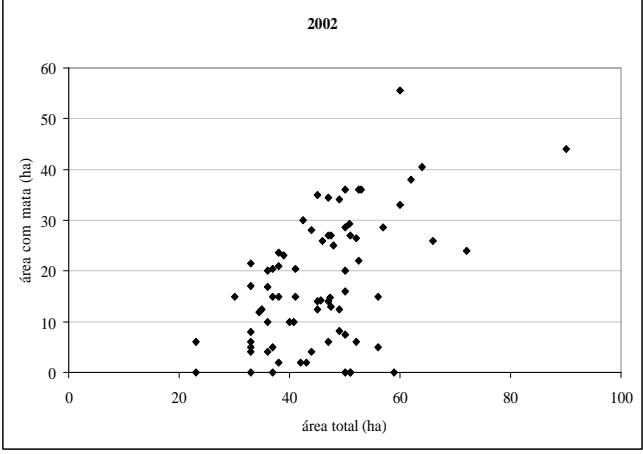

(b)

Figura 1 - Relação entre área total e área com mata para uma amostra de agricultores de Machadinho d'Oeste: (a) 1986; (b) 2002.

\subsection{Modelo}

Quanto à escolha do modelo DEA, neste artigo foi usado o modelo DEA BCC, já que não havia evidências de proporcionalidade entre inputs e outputs. Uma vez que o modelo CCR em sua forma fracionária apresenta uma função homogênea de grau zero, seria necessária 
esta proporcionalidade para justificar o seu uso. Para comprovar a não existência de proporcionalidade, basta observar a Figura 1. Nela fica evidente de que, embora haja resquícios de proporcionalidade entre área total e área com mata em 1986, esta é totalmente inexistente em 2002. Como era desejado verificar se a produção justificava a quantidade de recursos empregados, foi usada orientação a inputs. Na verdade, com esta orientação é possível verificar se há desperdícios de recursos na agricultura de Machadinho d’Oeste. Ressalte-se ainda que devido ao fato de a maioria da produção ser para consumo da família camponesa, o que caracteriza uma agricultura de subsistência, muitas vezes não há sequer interesse em aumentar os outputs.

Inicialmente foi calculado um modelo DEA para cada ano, com intuito de comparar os agricultores entre si, sem avaliação temporal. Em um segundo momento foi avaliada a evolução ao longo do tempo. Para tal existem dois modelos principais. O primeiro é com uso de índice de Malmquist (Färe et al., 1994), que é usado quando há intenção de avaliar a relação entre eficiência e mudança tecnológica, como o exemplo de Pereira et al. (2002). A outra abordagem é a de janelas de tempo (Cooper et al., 2000). Neste artigo foi usada uma versão simplificada desta última, em que cada produtor em um ano diferente é considerado como uma DMU diferente. Aplicações deste tipo podem ser vistas em Soares de Mello et al. (2003), Rios e Maçada (2006), por exemplo. Um outro contexto em que esta abordagem pode ser usada é quando há a necessidade de aumentar o numero de DMUs (Podinoviski \& Thanassoulis, 2007).

O modelo de sustentabilidade aqui proposto mede a capacidade de cada propriedade gerar receita e/ou preservar a mata nativa com os recursos que tem. É eficiente quem tem uma boa relação entre a receita e gastos, ou entre mão-de-obra e terra. Também é eficiente quem tiver uma boa relação entre mata preservada e gastos e/ou área total de terra da propriedade. Em casos extremos, pode haver unidades eficientes sem preservação ou sem produção. Estas situações limites só aparecem devido à possibilidade de serem atribuídos pesos nulos a algum output. Assim, para uma análise mais completa foram estudados dois enfoques: um que prioriza o aspecto econômico em relação ao ambiental e outro com prioridade oposta. Esses diferentes enfoques foram obtidos com a imposição de restrições aos pesos ARI ao modelo DEA BCC clássico (formulação (1) descrita anteriormente). No modelo de prioridade econômica foi imposto que o peso da variável receita bruta deveria ser maior que peso da variável área com mata. Já no modelo de prioridade ambiental, o peso de área com mata deveria ser maior que o peso de receita bruta. Isto corroborou com os diferentes enfoques de sustentabilidade, ou seja, pelo viés econômico a sustentabilidade parte do princípio da manutenção econômica do sistema de produção. Neste caso não importa muito o peso da preservação ambiental, cuja variável representativa pode ter peso nulo. Por outro lado, área de mata preservada não é contabilizada como serviço prestado ao sistema econômico e, neste caso, impôs-se peso maior para esta variável.

Neste ponto é importante destacar que ao impor restrições aos pesos do tipo ARI, caso o interesse seja medir a importância atribuída por cada DMU a cada variável, devem-se normalizar os dados. Evita-se, assim, que os valores resultantes dos pesos sejam influenciados pela escala dos dados. Entretanto, na proposta deste artigo, em relação ao modelo dos multiplicadores está-se interessado somente em avaliar a medida de eficiência e a quantidade de pesos zero atribuídos às variáveis. De qualquer forma, as variáveis foram normalizadas para evitar erros de interpretação dos resultados. 


\section{Resultados}

Conforme colocado anteriormente, foram adotadas duas abordagens para esse modelo, cujos resultados foram obtidos com o software SIAD (Angulo Meza et al., 2005). Segundo a abordagem de prioridade econômica, dos 76 lotes avaliados, 21 foram DEA-BCC eficientes em 1986 e 16 em 2002 (um modelo DEA para cada ano). Desses, seis foram eficientes em ambos os anos. Em 1986, a eficiência média foi de 0,7766; em 2002, 0,8190. Entre 1986 e 2002, 33 lotes $(43,4 \%)$ sofreram redução e 37 lotes $(48,7 \%)$ tiveram aumento de eficiência. A maior redução e o maior aumento foram, respectivamente, da ordem de $20,3 \%$ e $66,5 \%$. Os valores das medidas de eficiência encontram-se na Tabela 2. Na Tabela 3 verifica-se que houve aumento de eficiência média, aumento da mediana das eficiências e redução do desvio padrão. Estas três características em conjunto mostram uma situação de maior homogeneidade no conjunto de DMUs com o passar do tempo.

Tabela 2 - Medidas de eficiência segundo a abordagem de prioridade econômica.

\begin{tabular}{|c|c|c|c|c|c|}
\hline DMU & 1986 & 2002 & DMU & 1986 & 2002 \\
\hline DMU1 & 1,0000 & 0,8249 & DMU27 & 1,0000 & 1,0000 \\
\hline DMU2 & 0,5489 & 0,7307 & DMU28 & 1,0000 & 0,7350 \\
\hline DMU3 & 0,7338 & 0,5849 & DMU29 & 0,7805 & 1,0000 \\
\hline DMU4 & 0,8820 & 1,0000 & DMU30 & 1,0000 & 0,8713 \\
\hline DMU5 & 1,0000 & 1,0000 & DMU31 & 0,6707 & 1,0000 \\
\hline DMU6 & 1,0000 & 0,8914 & DMU32 & 0,6578 & 1,0000 \\
\hline DMU7 & 1,0000 & 0,8848 & DMU33 & 0,6835 & 0,7230 \\
\hline DMU8 & 0,7796 & 0,8926 & DMU34 & 0,5525 & 0,8439 \\
\hline DMU9 & 1,0000 & 0,9775 & DMU35 & 0,7296 & 0,9890 \\
\hline DMU10 & 0,6252 & 0,5990 & DMU36 & 0,9166 & 0,9903 \\
\hline DMU11 & 0,5353 & 0,7458 & DMU37 & 0,7472 & 0,5940 \\
\hline DMU12 & 1,0000 & 1,0000 & DMU38 & 0,6486 & 0,8024 \\
\hline DMU13 & 0,6538 & 0,5858 & DMU39 & 0,6283 & 0,5955 \\
\hline DMU14 & 0,6263 & 0,5764 & DMU40 & 0,6966 & 0,6748 \\
\hline DMU15 & 1,0000 & 0,8703 & DMU41 & 1,0000 & 0,6528 \\
\hline DMU16 & 0,6358 & 0,9212 & DMU42 & 0,7648 & 0,8120 \\
\hline DMU17 & 0,6475 & 0,7411 & DMU43 & 0,9435 & 0,9035 \\
\hline DMU18 & 0,7938 & 0,7624 & DMU44 & 0,7974 & 0,7652 \\
\hline DMU19 & 0,6836 & 0,6201 & DMU45 & 1,0000 & 1,0000 \\
\hline DMU20 & 1,0000 & 1,0000 & DMU46 & 0,6005 & 1,0000 \\
\hline DMU21 & 1,0000 & 0,8965 & DMU47 & 1,0000 & 0,9436 \\
\hline DMU22 & 0,9221 & 0,8236 & DMU48 & 0,7566 & 1,0000 \\
\hline DMU23 & 0,7021 & 0,6727 & DMU49 & 0,6305 & 0,8417 \\
\hline DMU24 & 0,5937 & 0,6366 & DMU50 & 0,7143 & 0,6888 \\
\hline DMU25 & 0,6291 & 1,0000 & DMU51 & 0,5369 & 0,7128 \\
\hline DMU26 & 1,0000 & 0,8498 & DMU52 & 0,6478 & 0,7500 \\
\hline
\end{tabular}

\begin{tabular}{ccc}
\hline DMU & 1986 & 2002 \\
\hline DMU53 & 0,6333 & 0,6527 \\
DMU54 & 1,0000 & 0,9970 \\
DMU55 & 1,0000 & 0,6253 \\
DMU56 & 0,7493 & 0,8225 \\
DMU57 & 0,6057 & 0,6566 \\
DMU58 & 0,6587 & 0,7442 \\
DMU59 & 0,7370 & 1,0000 \\
DMU60 & 0,7508 & 0,9003 \\
DMU61 & 0,7428 & 0,9328 \\
DMU62 & 0,6009 & 0,6408 \\
DMU63 & 0,6053 & 0,6837 \\
DMU64 & 0,7380 & 0,9108 \\
DMU65 & 0,7396 & 1,0000 \\
DMU66 & 0,6555 & 1,0000 \\
DMU67 & 1,0000 & 1,0000 \\
DMU68 & 0,7088 & 0,6343 \\
DMU69 & 0,6517 & 0,6547 \\
DMU70 & 0,8340 & 0,7356 \\
DMU71 & 0,6989 & 0,6029 \\
DMU72 & 0,6417 & 0,8745 \\
DMU73 & 1,0000 & 0,9418 \\
DMU74 & 0,5752 & 0,8277 \\
DMU75 & 1,0000 & 0,8892 \\
DMU76 & 0,6000 & 0,5372 \\
\hline & &
\end{tabular}


Tabela 3 - Resumo estatístico dos dados da Tabela 2.

\begin{tabular}{ccc}
\cline { 2 - 3 } & 1986 & 2002 \\
\hline Média & 0,7766 & 0,8190 \\
Mediana & 0,7375 & 0,8347 \\
Desvio padrão & 0,1606 & 0,1473 \\
N. $^{\circ}$ de DMUs eficientes & 21 & 16 \\
\hline
\end{tabular}

É importante ressaltar que, embora a eficiência média seja largamente usada na literatura (ver, por exemplo, Avkiram, 1999), seu aumento não significa melhora geral na avaliação das DMUs. Significa sim maior homogeneidade do conjunto de DMUs. Esta pode ter sido atingida tanto pela melhoria de desempenho das DMUs ineficientes, quanto pela piora de desempenho das unidades eficientes.

Nos resultados do modelo dos multiplicadores, em 1986, a variável de input que recebeu maior número de pesos zero (33,1\%) foi mão-de-obra. Já nos outputs, área com mata foi desconsidera por 18,4\% dos lotes. Em 2002, o input mão-de-obra e o output área com mata foram as variáveis que obtiveram o maior número de pesos zero (42,1\% em ambos os casos). Esses resultados indicam que, ao considerar que o aspecto econômico como mais importante que o ambiental no cálculo da medida de eficiência, o fator mais limitante para obtenção de eficiência na região é mão-de-obra.

Os resultados do modelo do envelope mostraram que o agricultor eficiente que apareceu o maior número de vezes como referência $(64,5 \%)$ para os demais foi, em 1986, um produtor alagoano, que chegou a Machadinho em 1985, e que tinha 83,9\% de área de mata natural e 3,8\% de área cultivada em relação à área total do lote. Para o ano de 2002, a DMU mais referenciada, com 65,8\% de contribuições não nulas, foi um produtor do ES, com chegada em Machadinho em 1984, cujo lote apresentava 77,8\% de área com mata natural. Recebeu assistência técnica da Emater, tinha empregados temporários e os ativos da família dedicavam-se parcialmente ao lote.

Segundo o enfoque da prioridade ambiental, conforme apresentado na Tabela 4, em 1986, 21 lotes (27,6\%) foram eficientes, com eficiência média de 0,9043. Já em 2002, a eficiência DEA BCC foi de 0,8063, com 17 DMUs (22,4\%) eficientes. Ainda, de 1986 a 2002, 50 lotes (65,8\%) reduziram o valor da eficiência, 21 (27,6\%) aumentaram e cinco (6,7\%) mantiveram o mesmo nível de eficiência (aqui, 1,0000). A maior redução foi de cerca de 76,8\% e o maior aumento de 49,6\%. Na Tabela 5 nota-se tendência oposta à do modelo anterior, ou seja, há redução de homogeneidade do conjunto de DMUs ao longo do tempo.

Os resultados do modelo dos multiplicadores mostraram que em 1986, o input ao qual foi atribuído o maior número de pesos zero $(84,2 \%)$ foi custo total. Para os outputs, a receita bruta recebeu peso nulo em 77,6\% dos lotes. Em 2002, a mão-de-obra (input) e a receita bruta (output) foram as variáveis com maior número de pesos zero (respectivamente, 46,1\% e 52,6\%). A redução na atribuição de pesos nulos para receita bruta indica que o aspecto econômico sofreu evolução positiva ao longo do período considerado. Em contrapartida, o número de pesos zero atribuídos pelas DMUs ao output área com mata, que passou de 11,8\% para $31,6 \%$, mostra que o aspecto ambiental pode ser limitante para a sustentabilidade desses produtores, o que corrobora com os resultados da abordagem anterior. 
Tabela 4 - Medidas de eficiência segundo o ponto de vista de prioridade ambiental.

\begin{tabular}{ccc}
\hline DMU & 1986 & 2002 \\
\hline DMU1 & 1,0000 & 0,8107 \\
DMU2 & 0,8216 & 0,7307 \\
DMU3 & 0,8101 & 0,5969 \\
DMU4 & 0,9194 & 1,0000 \\
DMU5 & 1,0000 & 1,0000 \\
DMU6 & 1,0000 & 0,8996 \\
DMU7 & 1,0000 & 0,9277 \\
DMU8 & 0,9475 & 0,8260 \\
DMU9 & 1,0000 & 1,0000 \\
DMU10 & 0,8431 & 0,5637 \\
DMU11 & 0,8485 & 0,7556 \\
DMU12 & 1,0000 & 1,0000 \\
DMU13 & 0,8570 & 0,5902 \\
DMU14 & 0,8216 & 0,5497 \\
DMU15 & 0,9935 & 0,8676 \\
DMU16 & 0,9318 & 0,9262 \\
DMU17 & 0,9389 & 0,6997 \\
DMU18 & 0,9015 & 0,7624 \\
DMU19 & 0,9973 & 0,6425 \\
DMU20 & 1,0000 & 1,0000 \\
DMU21 & 1,0000 & 0,9085 \\
DMU22 & 0,9547 & 0,8329 \\
DMU23 & 0,8455 & 0,6731 \\
DMU24 & 0,8447 & 0,6366 \\
DMU25 & 0,6684 & 1,0000 \\
DMU26 & 1,0000 & 0,8498 \\
\hline & &
\end{tabular}

\begin{tabular}{ccc}
\hline DMU & 1986 & 2002 \\
\hline DMU27 & 0,9471 & 1,0000 \\
DMU28 & 1,0000 & 0,7713 \\
DMU29 & 0,7826 & 1,0000 \\
DMU30 & 1,0000 & 0,8530 \\
DMU31 & 0,8696 & 1,0000 \\
DMU32 & 0,9657 & 1,0000 \\
DMU33 & 0,9600 & 0,7193 \\
DMU34 & 0,8545 & 0,8522 \\
DMU35 & 0,9050 & 0,9911 \\
DMU36 & 0,9679 & 0,9931 \\
DMU37 & 0,9188 & 0,5806 \\
DMU38 & 0,9450 & 0,8099 \\
DMU39 & 0,6805 & 0,5774 \\
DMU40 & 0,7658 & 0,6748 \\
DMU41 & 1,0000 & 0,6528 \\
DMU42 & 0,9610 & 0,8650 \\
DMU43 & 0,9435 & 0,9126 \\
DMU44 & 0,9996 & 0,8094 \\
DMU45 & 1,0000 & 0,2321 \\
DMU46 & 0,9165 & 1,0000 \\
DMU47 & 1,0000 & 0,9539 \\
DMU48 & 0,9648 & 1,0000 \\
DMU49 & 0,7516 & 0,8761 \\
DMU50 & 1,0000 & 0,6888 \\
DMU51 & 0,8600 & 0,7401 \\
DMU52 & 0,6985 & 0,7186 \\
\hline & &
\end{tabular}

\begin{tabular}{ccc}
\hline DMU & 1986 & 2002 \\
\hline DMU53 & 0,7992 & 0,6691 \\
DMU54 & 1,0000 & 1,0000 \\
DMU55 & 1,0000 & 0,6356 \\
DMU56 & 0,8822 & 0,8262 \\
DMU57 & 0,9149 & 0,6114 \\
DMU58 & 0,8570 & 0,7637 \\
DMU59 & 0,9171 & 1,0000 \\
DMU60 & 0,7967 & 0,8940 \\
DMU61 & 0,8432 & 0,9401 \\
DMU62 & 0,6418 & 0,6477 \\
DMU63 & 0,9238 & 0,6729 \\
DMU64 & 0,9076 & 0,8502 \\
DMU65 & 0,9076 & 1,0000 \\
DMU66 & 0,7819 & 1,0000 \\
DMU67 & 1,0000 & 1,0000 \\
DMU68 & 0,9048 & 0,6007 \\
DMU69 & 0,8660 & 0,5813 \\
DMU70 & 1,0000 & 0,7507 \\
DMU71 & 0,7199 & 0,6017 \\
DMU72 & 0,7397 & 0,8745 \\
DMU73 & 1,0000 & 0,7715 \\
DMU74 & 0,8293 & 0,8500 \\
DMU75 & 1,0000 & 0,8724 \\
DMU76 & 0,8929 & 0,5395 \\
\hline
\end{tabular}

Tabela 5 - Resumo estatístico dos dados da Tabela 4.

\begin{tabular}{ccc}
\cline { 2 - 3 } & 1986 & 2002 \\
\hline Média & 0,9043 & 0,8063 \\
Mediana & 0,9191 & 0,8295 \\
Desvio padrão & 0,0952 & 0,1636 \\
N.o de DMUs eficientes & 21 & 17 \\
\hline
\end{tabular}

É importante ainda ressaltar que na evolução temporal nos dois enfoques há uma redução da limitação à eficiência por conta dos custos e um aumento dessa limitação por conta da mãode-obra.

Pelo modelo do envelope verifica-se que a DMU eficiente que apareceu como benchmark mais referenciado pelas demais DMUs em 1986 (65,8\% das vezes), foi um produtor do Paraná, em Machadinho desde 1972, e que tinha 59,1\% do lote com mata natural. Em 2002, 
o agricultor mais referenciado (por $64,5 \%$ dos lotes) foi o mesmo produtor capixaba da abordagem anterior.

Destaca-se ainda que três produtores foram eficientes nos dois anos nas duas abordagens. Estas são as DMUs 5, 12 e 20. Para contribuir com a evolução do desempenho dos demais produtores e a manutenção ou o incremento de sua sustentabilidade, as práticas executadas por esses agricultores podem servir de subsídio para os demais (apoiados por órgãos de assistência técnica, extensão rural ou de pesquisa).

Estes agricultores chegaram a Machadinho d'Oeste entre 1973 e 1985, provenientes dos estados Rio Grande do Norte, Bahia e Minas Gerais. A área total de suas propriedades variava entre 23 e 45 ha. A área cultivada representava de 17 a 43\% da área total em 2002 e de 11 a $14 \%$ em 1986. A área com mata correspondia a um percentual entre 26 e $56 \%$ da área total em 2002 e de 73 a 89\% em 1986. Em 1986, um produtor recebeu assistência técnica da EMATER e usou o Núcleo Urbano de Assistência Rural (NUAR). Haviam contraído doença e a dedicação ao lote variava de 25 a $100 \%$ do tempo. Não usaram mão-deobra extrafamiliar. Dois tinham atividade extra-agrícola. Não participavam de sindicato ou de outra forma de organização social. Quanto à condição legal do lote, dois eram meeiros e um era parceiro. Um agricultor tinha casa de madeira; os demais, de pau-roliço. Apontaram na época saúde, estradas e falta de financiamento e de recursos humanos para propriedade como necessidade/problema. Em 2002, estes produtores rurais tinham entre 50 e 62 anos de idade. Dois deles não possuíam qualquer nível de instrução formal, enquanto que um havia concluído o $1^{\circ}$ grau. Um agricultor recebeu assistência técnica da EMATER e dois usaram o NUAR. Nenhum deles pensava em sair do lote, nem havia contraído doença. Dedicavam de 75 a 100\% de seu tempo ao lote. Não tinham empregado temporário ou permanente, nem atividade extra-agrícola. Dois deles haviam comprado o lote e um era posseiro. Todos possuíam casas de madeira. Indicaram moradia e estradas como necessidade/problema. Dois produtores participavam de grupos religiosos e um de sindicato.

Para verificar se o aumento de eficiência média constatado nos dois modelos corresponde realmente a uma melhoria de desempenho é necessária uma análise adicional. Esta análise consiste em comparar conjuntamente as unidades eficientes em cada um dos anos, usando um caso particular do modelo dinâmico de janelas de tempo (Cooper et al., 2000). Para tal, rodaram-se dois modelos DEA. Um, no enfoque de prioridade econômica, no qual foram usadas apenas as DMUs que foram eficientes neste modelo, em quaisquer dos dois anos considerados, em um total de 31 DMUs. De forma semelhante rodou-se um modelo com as 41 DMUs DEA eficientes no enfoque de prioridade ambiental. Em ambos os modelos foram mantidas as respectivas restrições aos pesos.

Ao compararem-se DMUs de anos diferentes em um mesmo modelo, as eficiências médias de grupos de DMUs passam a ter sentido absoluto. Como as DMUs usadas compunham as fronteiras dos modelos iniciais, a variação da média indica o sentido do deslocamento da fronteira eficiente.

No modelo conjunto de prioridade econômica, a eficiência média em 1986 foi de 0,9701 aumentando ligeiramente para 0,9728 em 2002 (13 cinco DMUs eficientes em um total de 21 em 1986, 12 DMUs eficientes em 2002 em um total de 16). No enfoque de prioridade ambiental, a eficiência média baixou de 0,9848 em 1986 para 0,9572 em 2002 (14 DMUs eficientes em um total de 21 em 1986, 10 DMUs eficientes em um total de 17 em 2002).

Enquanto que o modelo conjunto de prioridade ambiental sugere que as perdas de eficiência no modelo de anos separados são realmente perdas no modelo conjunto, o modelo do enfoque econômico não permite tirar quaisquer conclusões sobre o ganho de eficiência 
observado no modelo que avalia separadamente os dois anos em estudo. Isto se deve ao fato de que a eficiência média e o número de DMUs eficientes variaram em sentidos opostos e, mesmo assim, as variações foram muito pequenas.

Para desfazer esta ambiguidade foi rodado um novo modelo de enfoque econômico com todas as DMUs, ou seja, considerando um mesmo produtor em dois anos distintos como DMUs diferentes. Neste modelo, observou-se que as DMUs referentes ao ano de 1986 apresentaram eficiência média de 0,8105 e as referentes ao ano de 2002, 0,7127. Isto indica uma piora do desempenho econômico ao longo do tempo. Portanto, o aumento de eficiência média observado nos modelos separados era apenas resultante de aumento de homogeneidade no conjunto das DMUs.

A Figura 2 apresenta o gráfico da relação entre as variáveis área total e receita para as unidades eficientes na abordagem ambiental conjunta. Verifica-se neste gráfico que não existe proporcionalidade entre este par input-output para as unidades eficientes. Esta característica ratifica a hipótese inicial de que o modelo CCR não seria adequado.

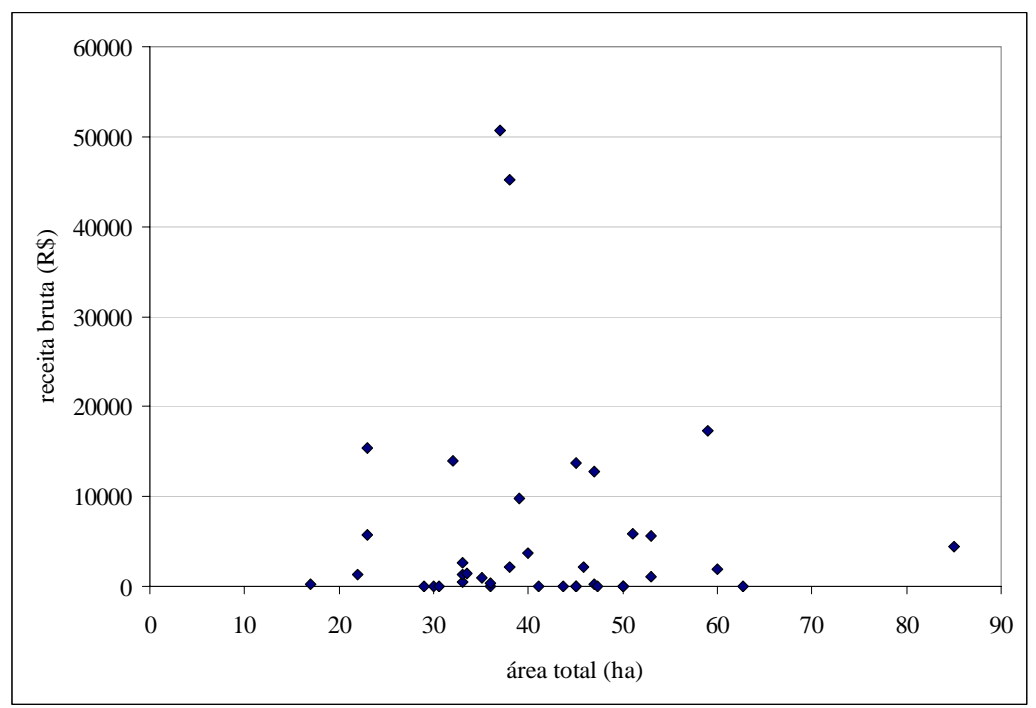

Figura 2 - Relação entre receita bruta e área total para as DMUs da abordagem conjunta segundo o ponto de vista ambiental.

\section{Conclusões}

Ao propor o uso de modelos DEA para avaliar a sustentabilidade em agricultura, este artigo contribui para o estado da arte no tema, em especial pelo fato de não ter sido encontrada referência na literatura de seu uso no caso brasileiro. A referência mais próxima é o trabalho de Toresan (1998), no qual a sustentabilidade é estudada com o método multicritério Macbeth e a eficiência com modelos DEA.

Os dois enfoques e a abordagem temporal aqui empregados possibilitaram uma visão mais ampla da situação dos agricultores na região. A análise temporal com a comparação das DMUs eficientes em um único modelo sugere que não há nem sustentabilidade do ponto de 
vista econômico nem do ambiental. Recomenda-se, assim, uma forte revisão das políticas públicas para a região.

A análise do modelo dos multiplicadores traz igualmente resultados importantes. Verificouse que a variável ambiental considerada (área com mata), mesmo quando sua importância relativa foi imposta superior à da renda bruta, é limitante para a sustentabilidade dos agricultores (pelo crescimento do número de pesos zero no segundo enfoque). No aspecto econômico, a variável mão-de-obra manteve-se como fator limitante.

Com a ampliação das áreas de culturas perenes (em especial café, e em menor intensidade cacau e guaraná) e de pastagens, houve aumento do desmatamento em cada propriedade em relação ao início do projeto de assentamento. $\mathrm{O}$ valor médio de área com mata em cada lote depende do tipo de exploração da propriedade rural ou, em outras palavras, da estrutura de produção. Em Machadinho d'Oeste, essa estrutura produtiva, tipicamente de lavouras e pecuária, é determinante e é determinada pelo uso das terras. A consolidação dos cultivos perenes, na medida em que sejam adotados alguns princípios de manejo mais ecológico, como o sombreamento com árvores nativas (principalmente para as culturas perenes) e a prática de restituições minerais, representará um elemento fundamental da sustentabilidade agrícola das propriedades rurais. Adicionalmente, conforme destacado em Mangabeira et al. (2005), a prática de sombreamento (especialmente para o café, principal cultura de renda de Machadinho) tem provado ser bastante eficiente, com contribuição para o aumento da produtividade (pela uniformidade de produção entre os anos, já que diminui o efeito bi-anual de safras altas e baixas), diminuição de pragas e doenças e restauração/manutenção de alguns nutrientes nos solos. Dessa forma, a prática de reflorestamento deve ser incentivada na região, o que pode e já vem sendo feito pelo plantio consorciado de seringueira e café. A extração de látex é pouco explorada devido ao baixo preço e, assim, os produtores cultivavam seringueira pelos benefícios proporcionados pelo sombreamento para a cultura de café, além de produzir maior conforto para os trabalhos manuais nos cafezais.

A identificação dos produtores eficientes, em especial daqueles que se mantiveram eficientes nos dois períodos de tempo e nos dois enfoques, pode ser útil na determinação de diretrizes de melhoria, por extensionistas, órgãos de assistência técnica e de pesquisa, aos demais produtores, em especial para aqueles que sofreram decréscimo no desempenho ao longo do tempo. Desenvolvimentos futuros devem focar a exploração dos perfis dos produtores de referência, já que estes garantiram, ao longo deste tempo, a manutenção dos seus sistemas de produção e a sustentabilidade econômico-ambiental desses sistemas.

\section{Agradecimentos}

Ao CNPq, pelo apoio financeiro. À Embrapa Monitoramento por Satélite, pelos dados.

\section{Referências Bibliográficas}

(1) Abay, C.; Miran, B. \& Gunden, C. (2004). An analysis of input use efficiency in tobacco production with respect to sustainability: The case study of Turkey. Journal of Sustainable Agriculture, 24(3), 123-143.

(2) Adler, N.; Friedman, L. \& Sinuany-Stern, Z. (2002). Review of ranking methods in the data envelopment analysis context. European Journal of Operational Research, 140, 249-265. 
(3) Ali, M. (1996). Quantifying the socio-economic determinants of sustainable crop production: an application to wheat cultivation in the Tarai of Nepal. Agricultural Economics, 14, 45-60.

(4) Allen, R.; Athanassopoulos, A.; Dyson, R.G. \& Thanassoulis, E. (1997). Weights restrictions and value judgements in data envelopment analysis: evolution, development and future directions. Annals of Operations Research, 73, 13-34.

(5) Angulo Meza, L. \& Lins, M.P.E. (2002). Review of methods for increasing discrimination in data envelopment analysis. Annals of Operations Research, 116, 225-242.

(6) Angulo Meza, L.; Biondi Neto, L.; Soares de Mello, J.C.C.B. \& Gomes, E.G. (2005). ISYDS - Integrated System for Decision Support (SIAD - Sistema Integrado de Apoio à Decisão): a software package for data envelopment analysis. Pesquisa Operacional, 25(3), 493-503.

(7) Avkiran, N.K. (1999). The evidence on efficiency gains: The role of mergers and the benefits to the public. Journal of Banking and Finance, 23(7), 991-1013.

(8) Banker, R.D.; Charnes, A. \& Cooper, W.W. (1984). Some models for estimating technical scale inefficiencies in data envelopment analysis. Management Science, 30(9), 1078-109.

(9) Barreto, R.C.S.; Khan, A.S. \& Lima, P.V.P.S. (2005). Sustentabilidade dos assentamentos do município de Caucaia-CE. Revista de Economia e Sociologia Rural, 43(2), 225-247.

(10) Batistella, M. (2001). Landscape change and land-use/land-cover dynamics in Rondônia, Brazilian Amazon. 2003. Thesis (Ph.D. dissertation), Indiana University, Bloomington, Indiana, 399 p.

(11) Batistella, M.; Brondizio, E.S. \& Moran, E.F. (2000). Comparative analysis of landscape fragmentation in Rondônia, Brazilian Amazon. International Archives of Photogrammetry and Remote Sensing and Spatial Information Sciences, 33, 148-155.

(12) Batistella, M.; Robeson, S. \& Moran, E.F. (2003). Settlement design, forest fragmentation, and landscape change in Rondônia, Amazônia. Photogrammetric Engineering and Remote Sensing, 69(7), 805-812.

(13) Battese, G.E. (1992). Frontier production functions and technical efficiency: a survey of empirical applications in agricultural economics. Agricultural Economics, 7, 185-208.

(14) Bosetti, V. \& Locatelli, G. (2006). A data envelopment analysis approach to the assessment of natural parks' economic efficiency and sustainability. The case of Italian national parks. Sustainable Development, 14(4), 277-286.

(15) Bravo-Ureta, B.E. \& Pinheiro, A.E. (1993). Efficiency analysis of developing country agriculture: a review of the frontier function literature. Agricultural and Resource Economics Review, 22, 88-101.

(16) Bravo-Ureta, B.E.; Solís, D.; López, V.H.M.; Maripani, J.F.; Thiam, A. \& Rivas, T. (2007). Technical efficiency in farming: a meta-regression analysis. Journal of Productivity Analysis, 27(1), 57-72.

(17) Carpenter, R.A. (1993). Can sustainability be measured?. Environmental Strategy, 5, 13-16.

(18) Castilla, R.E.F. \& Sawyer, D.O. (1993). Malaria rates and fate: A socioeconomic study of malaria in Brazil (1993). Social Science and Medicine, 37(9), 1137-1145. 
(19) Charlwood, J.D.; Alecrim, W.D.; Fe, N.; Mangabeira, J.A.C. \& Martins, V.J. (1996). A field trial with Lambda-cyhalothrin (ICON) for the intradomiciliary control of malaria transmitted by Anopheles darlingi root in Rondonia, Brazil (1996). Acta Tropica, 60(1), 3-13.

(20) Charnes, A.; Cooper, W.W. \& Rhodes, E. (1978). Measuring the efficiency of decisionmaking units. European Journal of Operational Research, 2, 429-444.

(21) Coelli, T.J. (1995). Recent developments in frontier modeling and efficiency measurement. Australian Journal of Agricultural Economics, 39(3), 219-245.

(22) Comissão Mundial sobre o Meio Ambiente e Desenvolvimento (1998). Nosso Futuro Comum. Fundação Getúlio Vargas, Rio de Janeiro, 8 p.

(23) Cooper, W.W.; Seiford, L.M. \& Tone, K. (2000). Data envelopment analysis: a comprehensive text with models, applications and DEA-solver software. Kluwer Academic Publishers, Boston.

(24) Dale, V.H.; O’Neill, R.V.; Southworth, F. \& Pedlowski, M. (1994). Modeling effects of land management in the Brazilian Amazon settlement of Rondônia. Conservation Biology, 8(1), 196-206.

(25) De Koeijer, T.J.; Wossink, G.A.A.; Struik, P.C. \& Renkema, J.A. (2002). Measuring agricultural sustainability in terms of efficiency: the case of Dutch sugar beet growers. Journal of Environmental Management, 66, 9-17.

(26) Edwards, C.A.; Lal, R.; Madden, P.; Miller, R.H. \& House, G. (1990). Sustainable Agricultural Systems. Soil and Water Conservation Society, Iowa, 1990.

(27) Ehlers, E. (1999). Agricultura Sustentável: origens e perspectivas de um novo paradigma. 2. ed., Ed. Agropecuária, Guaíba.

(28) Färe, R.; Grosskopf, S.; Norris, M. \& Zhang, Z. (1994). Productivity growth, technical progress, and efficiency change in industrialized countries. The American Economic Review, 84(1), 66-83.

(29) Fernandes, L.A.O. (2004). The meaning of sustainability: searching for agrienvironmental indicators. Thesis (Ph.D.) - University of Manchester. 332p.

(30) Gomes, E.G.; Soares de Mello, J.C.C.B. \& Mangabeira, J.A.C. (2008). Índice multicritério de bem estar social rural em um município da Região Amazônica. Pesquisa Operacional, 28(1), 141-160.

(31) Gomes, I. (2004). Sustentabilidade social e ambiental na agricultura familiar. Revista de Biologia e Ciências da Terra, 5(1).

(32) Gomes, E.G. (2008). Uso de modelos DEA em agricultura: revisão da literatura. Engevista, 10, 27-51.

(33) Herendeen, R.A. \& Wildermuth, T. (2002). Resource-based sustainability indicators: Chase County, Kansas, as example. Ecological Economics, 42, 243-257.

(34) Lins, M.P. \& Angulo Meza, L. (2000). Análise Envoltória de Dados e perspectivas de integração no ambiente de Apoio à Decisão. Editora da COPPE/UFRJ, Rio de Janeiro.

(35) Lins, M.P.E.; Gomes, E.G.; Soares de Mello, J.C.C.B. \& Soares de Mello, A.J.R. (2003). Olympic ranking based on a zero sum gains DEA model. European Journal of Operational Research, 148, 312-322.

(36) Lins, M.P.E.; Silva, A.C.M. \& Lovell, C.A.K. (2007). Avoiding infeasibility in DEA models with weight restrictions. European Journal of Operational Research, 181, 956-966. 
(37) Lopes, S.B. \& Almeida, J. (2003). Methodology for comparative analysis of sustainability in agroforestry systems. Revista de Economia e Sociologia Rural, 41(1), 183-208.

(38) López-Ridaura, S.; Masera, O. \& Astier, M. (2002). Evaluating the sustainability of complex socio-environmental systems: the MESMIS framework. Ecological Indicators, 2, 135-148.

(39) Mangabeira, J.A.C.; Miranda, E.E. \& Gomes, E.G. (2005). Perfil agrossocioeconômico dos produtores rurais de Machadinho d'Oeste (RO), em 2002. Documentos, 38. Embrapa Monitoramento por Satélite, Campinas, 114 p., il.

(40) Marzall, K. \& Almeida, J. (2000). Indicadores de sustentabilidade para agroecossistemas: estado da arte, limites e potencialidades de uma nova ferramenta para avaliar o desenvolvimento sustentável. Cadernos de Ciência \& Tecnologia, 17(1), 41-59.

(41) Melgarejo, L. (2000). Desempenho, eficiência multidimensional e previsão de possibilidade de sucesso em assentamentos de reforma agrária, no Rio Grande do Sul. Tese (Doutorado em Engenharia de Produção) - Universidade Federal de Santa Catarina. 482 p.

(42) Miranda, E.E. (2005). Sustentabilidade Agrícola na Amazônia: 20 anos de monitoramento da agricultura em Machadinho d'Oeste-RO. Embrapa Monitoramento por Satélite, Campinas, 1 CD-Rom.

(43) Moran, E.F. (1984). Amazon basin colonization. Interciencia, 9(6), 377-385.

(44) Pacini, C.; Wossink, A.; Giesen, G.; Vazzana, C. \& Huirne, R. (2003). Evaluation of sustainability of organic, integrated and conventional farming systems: a farm and field-scale analysis. Agriculture, Ecosystems and Environment, 95, 273-288.

(45) Parra-Lopez, C.; Calatrava-Requena, J. \& de-Haro-Gimenez, T. (2008). A systemic comparative assessment of the multifunctional performance of alternative olive systems in Spain within an AHP-extended framework. Ecological Economics, 64(4), 820-834.

(46) Pannell, D.J. \& Glenn, N.A. (2000). A framework for the economic evaluation and selection of sustainability indicators in agriculture. Ecological Economics, 33, 135-149.

(47) Pereira, M.F.; Silveira, J.S.T. \& Lanzer, E.A. (2002). Productivity growth and technological progress in the Brazilian agricultural sector. Pesquisa Operacional, 22(2), 133-146.

(48) Podinovski, V.V. \& Thanassoulis, E. (2007). Improving discrimination in data envelopment analysis: Some practical suggestions. Journal of Productivity Analysis, 28(1-2), 117-126.

(49) Praneetvatakul, S.; Janekarnkij, P.; Potchanasin, C. \& Prayoonwong, K. (2001). Assessing the sustainability of agriculture: a case of Mae Chaem Catchment, northern Thailand. Environment International, 27, 103-109.

(50) Pretty, J. (2008). Agricultural sustainability: concepts, principles and evidence. Philosophical Transactions of the Royal Society B-Biological Sciences, 363(1491), 447-465.

(51) Rasul, G. \& Thapa, G.B. (2004). Sustainability of ecological and conventional agricultural systems in Bangladesh: an assessment based on environmental, economic and social perspectives. Agricultural Systems, 79, 327-351.

(52) Rigby, D.; Woodhouse, P.; Young, T. \& Burton, M. (2001). Constructing a farm level indicator of sustainable agricultural practice. Ecological Economics, 39, 463-478.

(53) Rios, L.R. \& Maçada A.C. (2006). Analysing the relative efficiency of container terminals of Mercosur using DEA. Maritime Economics \& Logistics, 8, 331-346. 
(54) Rodríguez-Díaz, J.A.; Camacho-Poyato, E. \& López-Luque, R. (2004). Application of data envelopment analysis to studies of irrigation efficiency in Andalusia. Journal of Irrigation and Drainage Engineering, 130(3), 175-183.

(55) Sachs, I. (1990). Desarrollo sustentable, bio-industrialización descentralizada y nuevas configuraciones rural-urbanas: los casos de India y Brasil. Pensamiento Iberoamericano, 46, 235-256.

(56) Sachs, I. (2000). Caminhos para o desenvolvimento sustentável. Ed. Garamond, Rio de Janeiro.

(57) Sauer, J. \& Abdallah, J.M. (2007). Forest diversity, tobacco production and resource management in Tanzania. Forest Policy and Economics, 9(5), 421-439.

(58) Senra, L.F.A.C.; Gonçalves, L.C.N.I.; Soares de Mello, J.C.C.B. \& Angulo Meza, L. (2007). Estudo sobre métodos de seleção de variáveis em DEA. Pesquisa Operacional, 27(2), 191-207.

(59) Soares de Mello, J.C.C.B.; Angulo Meza, L.; Gomes, E.G.; Serapião, B.P. \& Lins, M.P.E. (2003). Análise de Envoltória de Dados no estudo da eficiência e dos benchmarks para companhias aéreas brasileiras. Pesquisa Operacional, 23(2), 325-345.

(60) Souza-Santos, R. (2002). Seasonal distribution of malaria vectors in Machadinho d'Oeste, Rondônia State, Amazon Region, Brazil. Cadernos de Saúde Pública, 18(6), 1813-1818.

(61) Sydenstricker, J.M. \& Torres, H.G. (1991). Mobility of migrants: autonomy or subordination in the Amazon region?. Revista Brasileira de Estudos de População, 8(1-2), 33-54.

(62) Thanassoulis, E.; Portela, M.C.S. \& Allen, R. (2004). Incorporating value judgments in DEA. In: Handbook on data envelopment analysis [edited by W.W. Cooper, L.M. Seiford \& J. Zhu], Kluwer Academic Publishers, Boston.

(63) Thiam, A.; Bravo-Ureta, B.E. \& Rivas, T.E. (2001). Technical efficiency in developing country agriculture: a meta-analysis. Agricultural Economics, 25, 235-243.

(64) Thompson, R.G.; Langemeier, L.N.; Lee, C.T.; Lee, E. \& Thrall, R.M. (1990). The role of multiplier bounds in efficiency analysis with application to Kansas farming. Journal of Econometrics, 46, 93-108.

(65) Toresan, L. (1998). Sustentabilidade e desempenho produtivo na agricultura: uma abordagem multidimensional aplicada a empresas agrícolas. Tese (Doutorado em Engenharia de Produção) - Universidade Federal de Santa Catarina.

(66) Veiga, J.E. (1996). Agricultura familiar e sustentabilidade. Cadernos de Ciência \& Tecnologia, 13(3), 383-404.

(67) Von Wirén-Lehr, S. (2001). Sustainability in agriculture - an evaluation of principal goal-oriented concepts to close the gap between theory and practice. Agriculture, Ecosystems and Environment, 84, 115-129.

(68) Zhen, L. \& Routray, J.K. (2003). Operational indicators for measuring agricultural sustainability in developing countries. Environmental Management, 32(1), 34-46. 